\title{
Neoplasia intra-epitelial cervical: diagnóstico, tratamento e seguimento em uma unidade básica de saúde
}

Cervical intraepithelial neoplasia: diagnosis, treatment and follow-up in a primary care unit

Luís Carlos Machado Junior ${ }^{1,2}$, Ana Sílvia Whitaker Dalmaso ${ }^{1}$

\section{Resumo}

Objetivo: Apresentar os resultados da atenção a pacientes com citologia oncótica vaginal anormal em unidade básica de saúde. Casuística e Métodos: Avaliação de mulheres com citologia anormal, por meio de colposcopia e biópsia, como parte do atendimento rotineiro da unidade integrada a serviço de referência. As pacientes com biópsia mostrando neoplasia intra-epitelial cervical (NIC) I, NIC II, cervicite, HPV ou colposcopia normal foram mantidas na unidade básica e tratadas quando indicado. Resultados: Em pouco mais de dez anos, 115 mulheres foram incluídas na série. Na primeira avaliação, 13 tiveram NIC III à biópsia e foram encaminhadas. As 102 pacientes restantes foram seguidas por um período médio de 38 meses; entre elas duas evoluíram para NIC III, ambas com biópsia inicial mostrando NIC II. Conclusão: 0 estudo sugere que é possível realizar esse tipo de atendimento em uma unidade básica com segurança aceitável, desde que a mesma disponha da estrutura mínima que é descrita no texto.

Unitermos: Displasia do colo uterino; neoplasias do colo do útero; atenção primária à saúde; sistemas de assistência à saúde; programação.

\begin{abstract}
Objective: The purpose of this study was to relate the results of the care of women with abnormal cervical cytology in a primary care unit. Subjects and Methods: Women with abnormal cytology were evaluated with colposcopy and, if necessary, biopsy, as part of the routine care of the unit. The cases in which biopsy showed cervical intra-epithelial neoplasia (CIN) III were referred to a specialized unit, and the remainder (CIN I, II and other results) were kept in the unit and treated when indicated. Results: In ten and half years, 115 women were included in the series. In the first biopsy, 13 had CIN III and were then referred. The remainder 102 were followed for an average period of 38 months. Two of them progressed to CIN III, both initially CIN II. Conclusion: This series suggests that this kind of care can be given in a primary care unit, provided this unit has the minimum resources described in the text.
\end{abstract}

Keywords: Uterine cervical dysplasia; uterine cervical neoplasms; primary health care; delivery of health care; programming.

Recebido: 09/05/2007

Revisado: 09/01/2008

Aprovado: $11 / 01 / 2008$

Trabalho realizado no Centro de Saúde-Escola Samuel Barnsley Pessoa (CSE Butantã), São Paulo (SP), Brasil.

Centro de Saúde-Escola Samuel Barnsley Pessoa, Faculdade de Medicina da Universidade de São Paulo (USP), São Paulo (SP), Brasil.

2 Disciplina de Ginecologia e Obstetrícia da Faculdade de Medicina do ABC (FMABC), São Paulo (SP), Brasil.

Endereço para correspondência: Luís Carlos Machado Junior, Avenida Doutor Vital Brasil, 1490 - Butantã, CEP 05503-000 - São Paulo (SP),

Tel.: (11) 3726-3305/8452, Fax: (11) 3726-1690. E-mail: csesbp@usp.br 


\section{Introdução}

No atendimento ambulatorial praticado no setor público, a coleta rotineira da citologia oncótica vaginal, ou citologia de Papanicolau, já foi incorporada à rotina. Em grande parte das unidades de atendimento, houve inclusive, a preocupação de desvincular a coleta do exame, da consulta médica, oferecendo coleta feita pela enfermagem para as mulheres que não necessitam atendimento médico, possibilitando que o exame seja realizado para um número maior de mulheres, aumentando assim a cobertura da população. Quando se obtém um resultado alterado, a prática tradicional tem sido encaminhar as pacientes para unidades de referência, para confirmação do diagnóstico, que normalmente é feita com colposcopia e biópsia, e para eventual tratamento. Essas unidades são normalmente serviços ambulatoriais com maior disponibilidade de recursos, hospitais ou centros universitários.

Este trabalho pretende apresentar a experiência de seguimento das pacientes que revelaram alteração à citologia oncótica, no Centro de Saúde Escola Professor Samuel Barnsley Pessoa, que é uma unidade básica de saúde (UBS), no período de 1995 a 2006. Nessas pacientes, foram feitas coleta, avaliação diagnóstica, tratamento e seguimento na referida instituição.

Vários autores se preocuparam em avaliar o risco de evolução das displasias leves e moderadas do colo uterino para formas mais avançadas (neoplasia intra epitelial grau III, displasia acentuada, carcinoma in situ ou lesão intra epitelial cervical de alto grau, segundo as várias nomenclaturas) ou para o carcinoma invasor. Para facilitar a exposição, adotaremos a seguir as expressões neoplasia intra-epitelial cervical grau I, II e III (NIC I, NIC II e NIC III) ${ }^{1-2}$ para nos referirmos a estas lesões.

A literatura apresenta valores bastante discrepantes ${ }^{3}$ quanto à magnitude deste risco, cuja avaliação apresenta uma série de dificuldades. Em primeiro lugar, este tem relação com o tempo de seguimento. Além disso, a maior parte das instituições realiza o tratamento assim que é feito o diagnóstico, o que é compreensível, levando-se em conta que a princípio são consideradas lesões pré-cancerosas. Esta conduta, porém, dificulta o conhecimento da história natural dessas condições.

Acreditamos que outra provável causa para estas diferenças seja a seguinte: grande parte das séries é de pacientes com diagnóstico baseado apenas em citologia. Considera-se, a princípio, o exame histológico, usualmente obtido por biópsia dirigida por colposcopia, como o padrão ouro para o diagnóstico dessas lesões. É bem conhecida a possibilidade de a citologia subestimar (ou superestimar) a gravidade de uma lesão, ou seja, de não haver uma perfeita correlação citológico/histológica. Desse modo, algumas lesões incluídas nas séries como lesões de baixo grau (NIC I) com base na citologia já seriam inicialmente lesões mais avançadas, e quando se constata esta lesão mais avançada, interpretase como evolução. Além disso, sabe-se que a biópsia do colo, além de fornecer informação diagnóstica, pode provocar uma reação local que contribui para a regressão da lesão ${ }^{4}$. Poderíamos supor, que as séries nas quais o diagnóstico é baseado no exame histológico, teriam uma taxa menor de evolução. Isto é o que se observa de fato. Na extensa revisão da literatura feita por Östör ${ }^{3}$, publicada em 1993, a composição dos dados de todas as séries (com diferentes períodos de acompanhamento e sem tratamento) mostra um risco médio de evolução de NIC I para NIC III de $11 \%$, e de $1 \%$ para carcinoma invasor. Porém, numa série mais recente que este autor apresenta, com diagnóstico baseado em biópsia, a taxa de evolução para NIC III foi de $2 \%$. Ainda segundo Östör, em relação à NIC II, a composição dos dados mostra um risco de evolução para NIC III de 23\%, e de 5\% para carcinoma invasor, incluindo séries com diagnóstico por biópsia ou citologia.

Em relação a estudos posteriores, Lee $(1998)^{5}$ adotou conduta expectante em 53 mulheres com diagnóstico de NIC I confirmado por biópsia. Teve dois casos (3,8\%) com evolução para NIC III com 34 e 48 meses de seguimento e nenhum caso com evolução para carcinoma invasor. Falls $(1999)^{6}$, em 89 pacientes de clínica privada com diagnóstico confirmado por biópsia e também com conduta expectante, teve, após seguimento de um ano, um caso $(1,1 \%)$ de evolução para NIC II e nenhum caso de evolução para NIC III ou para carcinoma invasor. Em nosso meio, Cardial (2001) ${ }^{7}$ acompanhou com conduta expectante, 79 pacientes com diagnóstico de NIC I confirmado por biópsia, pelo período de um ano. Teve quatro casos $(5,1 \%)$ de evolução para NIC II e nenhuma evolução para NIC III ou carcinoma invasor.

Alguns autores propõem a utilização rotineira da pesquisa e identificação dos subtipos de papilomavírus humano (HPV) nas pacientes com citologia alterada, para identificar quais necessitariam colposcopia ou para melhor individualização do tratamento e seguimento, uma vez feito o diagnóstico de NIC ${ }^{8-12}$.

Não encontramos na literatura médica brasileira relatos de experiências semelhantes à que estamos apresentando (seguimento em UBS). Na literatura internacional, a que mais se aproxima é a experiência de clínicas de medicina de família nos Estados Unidos ${ }^{13-14}$. Um relato de 1992 apresenta $200 \operatorname{casos}^{13}$, dos quais 102 tinham alteração citológica e os demais eram mulheres de risco para NIC. Foram encaminhadas 28 pacientes (14\%) para unidades de referência. O relato mais recente ${ }^{14}$, de 2000, mostra série de 283 mulheres com citologia alterada. Deste total, apenas $26(9 \%)$ foram encaminhadas, devido principalmente a lesões extensas ou que precisavam de conização. Em ambos os relatos, porém, a unidade em que se realizava a colposcopia ficava em local diferente de onde era realizado o acompanhamento clínico.

\section{Casuística e Métodos}

A casuística apresentada foi de pacientes atendidas regularmente no Centro de Saúde Escola Professor Samuel Barnsley Pessoa, ligado à Faculdade de Medicina da Universidade de São Paulo.

Descreveremos então a rotina de trabalho na unidade. As pacientes que freqüientam a unidade são orientadas por todos os profissionais de 
saúde para a coleta regular de citologia de Papanicolau. A coleta é feita por vários profissionais, aumentando a oportunidade de cuidado, tanto pela enfermagem, como em consulta médica. A partir de uma anotação no cadastro individual de saúde incluído no prontuário, é indicada para cada mulher a periodicidade de controle do câncer ginecológico, conforme preconizado pelo Ministério da Saúde ${ }^{15}$. No período indicado, a coleta é feita pelo profissional que está fazendo o acompanhamento da paciente (atividade de enfermagem ou médica, com médico generalista ou ginecologista), buscando-se otimizar os recursos e ampliar a cobertura da população. No primeiro ano de seguimento desta série, de 1995 a 1996, utilizava-se a coleta do fundo de saco e exocérvice. A partir de 1996, tornou-se rotineira a coleta tríplice, ou seja, fundo de saco, exocérvice e endocervical com escova, exceto para gestantes, das quais não se colhe material endocervical. Apesar de atualmente na unidade ser realizada apenas a coleta da exocérvice e endocervical, até o final do período em que os dados foram incluídos neste estudo (abril de 2006), era rotineira a coleta tríplice. Após dois exames normais com intervalo de um ano, propõe-se intervalo de três anos entre as coletas ${ }^{15-16}$, desde que estes se mantenham normais. Nos dois primeiros anos de seguimento desta casuística (1995 e 1996), o intervalo proposto era de dois anos.

O ginecologista atende casos triados pelo restante da equipe, sendo que alteração de citologia oncótica é uma das várias situações nas quais se encaminha pacientes para este especialista. Algumas pacientes tiveram a alteração citológica detectada no próprio seguimento ginecológico, por outras condições.

Os critérios para encaminhamento de alterações citológicas para o ginecologista são: NIC I, II e III, alterações compatíveis com ação do HPV e "atipias de significado indeterminado" de células escamosas (ASCUS) ou de células glandulares (AGUS). No período estudado, o laboratório de citologia não diferenciava os achados de atipias em "células escamosas atípicas de significado indeterminado" (ASC-US) de "células escamosas atípicas de significado indeterminado - não se pode excluir lesão de alto grau” (ASC-H), como recomendado pela nomenclatura de Bethesda de $2001^{17}$, o que poderia diferenciar a conduta em cada um dos tipos ${ }^{18}$. Os raros casos com citologia sugestiva de carcinoma invasor ou de atipias glandulares (AGUS) são encaminhados diretamente para o serviço de referência. Durante o período estudado, porém, não houve citologia sugestiva de nenhuma dessas duas últimas condições.

As pacientes com estas alterações são submetidas a uma consulta ginecológica padrão (com anamnese e exame físico) e, no mesmo ato, à colposcopia e, se necessário, biópsia dirigida do colo uterino com pinça de saca bocado. O exame anatomopatológico é realizado pelo Hospital Universitário da Universidade de São Paulo, que é um dos hospitais de referência da unidade.

Frente ao resultado da biópsia, temos o seguinte protocolo: as pacientes com NIC III (13 casos na série) ou carcinoma invasor (nenhum caso) são encaminhadas ao serviço de referência. As pacientes com os demais diagnósticos (NIC I, NIC II, alterações sugestivas de infecção por HPV e cervicite crônica) são mantidas em seguimento na unidade. Propomos tratamento para NIC I, NIC II e HPV. Durante o tempo do estudo, as mulheres com diagnóstico histológico de cervicite crônica foram mantidas em seguimento com intervalo proposto de seis meses. O tratamento proposto para as outras três condições acima foi a cauterização do colo uterino com eletrocautério, sob visão colposcópica. São cauterizadas apenas as áreas colposcopicamente alteradas. Não realizamos cauterização de ectopia que eventualmente exista ${ }^{19}$. Se no momento da cauterização não for encontrada alteração colposcópica, não realizamos o procedimento. Apesar de haver questionamento sobre o benefício do tratamento rotineiro das NIC I, decidimos realizá-lo por considerarmos uma conduta mais prudente, por se tratar de uma situação nova de seguimento e tratamento.

Após o tratamento inicial, agendamos um retorno entre três ou quatro meses para verificar o resultado do tratamento. Este é avaliado novamente com citologia, colposcopia e biópsia se necessário. Quando existe persistência da condição, o tratamento é repetido. Durante o seguimento, em nenhuma paciente foi necessário realizar mais do que duas cauterizações. O seguimento proposto para as pacientes "curadas" ou que não necessitaram tratamento foi de colposcopia e citologia a cada seis meses, do mesmo modo que para as pacientes com diagnóstico inicial de cervicite. Muitas pacientes não cumprem o seguimento proposto. As pacientes deste grupo são mantidas em um sistema de vigilância, que acompanha o comparecimento para os retornos preconizados e convoca faltosos. As convocações são feitas por carta ou telefonema. Quando não se consegue que a paciente retorne por estes meios, realiza-se visita domiciliar para conversar com ela sobre a importância do acompanhamento. Consegue-se reverter grande parte dos abandonos de seguimento por estes meios.

Começamos a seguir na unidade as pacientes com citologia alterada, com a sistemática descrita acima, a partir de setembro de 1995. Tomamos esta decisão levando em conta o risco relativamente pequeno de evolução para formas mais graves a partir das NIC II e principalmente das NIC I, apontado na revisão exposta acima. Para acompanhar os casos há um sistema de vigilância à saúde. Os prontuários são marcados e há um arquivo para registro do seguimento, detecção de faltas e providências. Na série que apresentaremos a seguir, incluímos todas as pacientes que tiveram, a partir daquela data, citologia sugestiva de NIC I, II e III, além de pacientes com outros resultados de citologia, mas que tiveram NIC diagnosticada por colposcopia e biópsia. Não incluímos na série pacientes que tiveram citologia mostrando ASCUS, AGUS ou sugestivas de HPV, nas quais não se encontrou NIC à biópsia, embora elas tenham tido um acompanhamento idêntico ao daquelas que apresentaram NIC à citologia.

A unidade conta com um sistema informatizado de armazenamento de dados para vigilância e avaliação. Os dados sobre citologias alteradas são, assim como dados sobre biópsias, rotineiramente registrados nesse sistema. Rastreamos as pacientes da série por meio da consulta a este sistema, cruzada com consulta ao livro de registro de biópsias. No início 
do período do estudo, as pacientes com citologias mostrando ASCUS ou HPV não eram incluídas no sistema de vigilância (as citologias mostrando ASCUS eram pouco freqüentes no período). Por este motivo, como foi colocado acima, as pacientes com esses resultados de citologia foram incluídas na série apenas quando a biópsia mostrou NIC. Quando se atendia pacientes que se enquadravam nos critérios de inclusão descritos acima, verificava-se se de fato elas constavam do sistema de vigilância. Os dados foram colhidos dos prontuários das pacientes deste modo rastreadas.

\section{Resultados}

No período de agosto de 1995 a abril 2006, entraram na série 115 pacientes. Destas, 114 não tinham história anterior de citologia alterada. Durante esse período, foram colhidas na unidade 13206 citologias. A idade média das pacientes ao entrar na série foi de 28,4 anos, variando de 16 a 58 anos. Os resultados serão apresentados segundo dois aspectos: a correlação citológico/histológica e a evolução.

a) Correlação citológico/histológica :

A Tabela 1 mostra a distribuição das pacientes quanto ao resultado da citologia.

A Tabela 2 mostra a correlação da histologia nas pacientes com NIC I à citologia.

Observou-se que nas pacientes com NIC I à citologia, houve predomínio (64,9\%) de achado histológico/colposcópico menos avançado que à citologia, 20,8\% com concordância e 14,3\% de achado mais avançado à primeira avaliação.

Houve 20 casos com NIC II à citologia. A correlação com a histologia é mostrada na Tabela 3.

Das cinco pacientes com NIC III à citologia, três apresentaram resultado concordante à biópsia e duas tiveram como resultado cervicite + HPV.

Das onze pacientes da série com citologia mostrando ASCUS, seis tiveram biópsia mostrando NIC I, uma NIC II e quatro NIC III. Deve-se ressaltar, contudo, que estas pacientes foram incluídas na série devido ao resultado da biópsia. Como relatado no Método, na unidade várias outras pacientes tiveram este resultado citológico (ASCUS) e não apresentaram NIC à avaliação rotineira (colposcopia e biópsia se necessário). Estas não foram incluídas na série por não estarem de acordo com os critérios de inclusão adotados. Como relatado acima, não houve no período pacientes com citologia sugestiva de atipias glandulares (AGUS) ou de carcinoma invasor.

Duas pacientes foram incluídas na série apesar de apresentarem citologia normal. Uma teve NIC I detectada em biópsia feita em colposcopia indicada por achado clínico de teste de Schiller positivo, e outra teve também NIC I detectada por biópsia em colposcopia, indicada por história pregressa de citologia alterada, em outra instituição.

b) Evolução

O tempo de evolução foi contado a partir da data da primeira citologia alterada, pois este achado foi o critério básico para a inclusão na série. Foram exceção as duas únicas pacientes da série com citologia normal, nas quais se contou a evolução a partir da data da biópsia.

Tivemos 34 pacientes com NIC I confirmado histologicamente, incluindo seis pacientes cuja biópsia inicial mostrou cervicite e durante o seguimento uma nova biópsia mostrou NIC I. Do total de 34 pacientes, cinco não retornaram após a biópsia e uma não retornou após o tratamento. As 28 pacientes restantes foram acompanhadas por um período médio de 42,1 meses, variando de nove a 111 meses. Deste grupo, 11 pacientes não foram tratadas, por motivos variados. Destas 11, acompanhadas por um período médio de 40,6 meses, duas (18,7\%) evoluíram para NIC II, uma após 18 e outra após 34 meses da citologia inicial. As outras nove estavam livres de neoplasia na última avaliação. As 17 pacientes tratadas foram acompanhadas por um período médio de 42,7 meses. Duas (11,8\%) evoluíram para NIC II, uma após 38 e outra após 58 meses. Das 15 restantes, uma apresentava ainda NIC I após 19 meses de seguimento e as demais não apresentavam neoplasia. Em toda a série, não houve evolução de NIC I para NIC III.

Tabela 1 - Distribuição das 115 pacientes da série estudada quanto ao resultado da citologia

\begin{tabular}{lrc}
\hline Resultado da citologia & N & $\begin{array}{c}\text { Proporção (\%) } \\
\text { com diagnóstico }\end{array}$ \\
\hline NIC I & 77 & 66,9 \\
NIC II & 20 & 17,4 \\
NIC III & 5 & 4,3 \\
ASCUS & 11 & 9,6 \\
Normal & 2 & 1,7 \\
Total & 115 & 100,0 \\
\hline
\end{tabular}

Tabela 2 - Distribuição do resultado do exame histológico em 77 pacientes com NIC I na citologia

\begin{tabular}{lcc}
\hline $\begin{array}{l}\text { Resultado do exame } \\
\text { histológico }\end{array}$ & N & $\begin{array}{c}\text { Proporção (\%) do } \\
\text { diagnóstico }\end{array}$ \\
\hline Colposcopia normal $^{\text {a }}$ & 15 & 19,48 \\
\hline Cervicite & 26 & 33,77 \\
Cervicite + HPV & 10 & 12,99 \\
NIC I & 16 & 20,78 \\
NIC II & 6 & 7,79 \\
NIC III & 4 & 5,19 \\
Total & 77 & 100,00
\end{tabular}

a Uma vez que a colposcopia não mostrou alterações, não foi feita biópsia e, portanto, não foi feito exame histológico.

Tabela 3 - Distribuição do resultado do exame histológico em 20 pacientes com NIC II na citologia

\begin{tabular}{lcc}
\hline $\begin{array}{l}\text { Resultado do exame } \\
\text { histológico }\end{array}$ & N & $\begin{array}{c}\text { Proporção (\%) } \\
\text { do diagnóstico }\end{array}$ \\
\hline Colposcopia normal $^{\mathrm{a}}$ & 2 & 10 \\
\hline Cervicite & 6 & 30 \\
NIC I & 4 & 20 \\
NIC II & 6 & 30 \\
NIC III & 2 & 10 \\
Total & 20 & 100 \\
\hline
\end{tabular}

a Uma vez que a colposcopia não mostrou alterações, não foi feita biópsia e, portanto, não foi feito exame histológico. 
Tabela 4 - Evolução de pacientes com NIC I e NIC II

\begin{tabular}{|c|c|c|}
\hline & Variável & $\mathrm{N}$ \\
\hline \multirow[t]{10}{*}{ NIC I } & Abandono do seguimento & 6 \\
\hline & Acompanhadas (período médio de 42,1 meses) & 28 \\
\hline & Não tratadas por motivos variados & 11 \\
\hline & Evolução para NIC II & 2 \\
\hline & Regressão & 9 \\
\hline & Tratadas & 17 \\
\hline & Evolução para NIC II & 2 \\
\hline & Persistência como NIC I & 1 \\
\hline & Regressão & 14 \\
\hline & Total & 34 \\
\hline \multirow[t]{9}{*}{ NIC II } & Abandono do seguimento & 3 \\
\hline & Acompanhadas (período médio de 37,8 meses) & 13 \\
\hline & Não tratadas por motivos variados & 2 \\
\hline & Persistência como NIC II & 1 \\
\hline & Regressão & 1 \\
\hline & Tratadas & 11 \\
\hline & Evolução para NIC III & 2 \\
\hline & Regressão & 9 \\
\hline & Total & 16 \\
\hline
\end{tabular}

Dezesseis casos de NIC II foram confirmados histologicamente, incluindo quatro pacientes que evoluíram a partir de NIC I. Duas pacientes não retornaram após a biópsia e uma não retornou após o tratamento. As 13 restantes foram acompanhadas por um período médio de 37,8 meses, variando de sete a 85 meses. Duas pacientes não foram tratadas: a primeira abandonou o seguimento e, em retorno recente, à biópsia apresentou novamente NIC II, 20 meses após a citologia inicial. A segunda, no retorno para tratamento, não apresentava mais lesão à colposcopia, também recentemente. Deste grupo de 13 pacientes, houve duas $(15,4 \%)$ que evoluíram para NIC III. A primeira paciente foi tratada e houve regressão. Decorridos 49 meses da citologia inicial, em exame colposcópico de rotina, apresentou NIC III à biópsia, foi, então, encaminhada ao serviço de referência e submetida à conização. Já teve alta do serviço de referência e está novamente em seguimento na unidade, livre de neoplasia. A segunda paciente foi tratada, teve também regressão e em colposcopia rotineira apresentou biópsia com NIC III 29 meses após a citologia inicial; está em seguimento na unidade de referência e já foi submetida à conização. Das demais 11 pacientes que permaneceram na unidade (13 menos duas que evoluíram para NIC III), havia persistência de NIC II em duas (uma delas não tratada, como descrito acima) e nove estavam livres de neoplasia por ocasião da última avaliação. A síntese das evoluções é mostrada na Tabela 4.

Treze casos tiveram diagnóstico histológico inicial de NIC III (11,3\% do total), além das duas descritas acima que evoluíram a partir de NIC II. Essas pacientes, segundo o programa estabelecido na unidade, não foram tratadas no serviço, e, portanto, não temos dados completos sobre o seguimento. Dessas 13 pacientes, apenas três (23\%) tinham citologia concordante (NIC III). Das dez restantes, quatro tinham citologia mostrando ASCUS, quatro com citologia sugestiva de NIC I e duas de NIC II.

Houve 56 pacientes ( $48,7 \%$ do total) nas quais, ao longo do seguimento, não se confirmou NIC à avaliação colposcópica/histológica.

Das 102 pacientes que foram seguidas em nosso serviço (115 menos 13 com diagnóstico inicial de NIC III), houve apenas duas (1,96\%) evoluções para NIC III (casos descritos acima). O tempo médio de seguimento destas 102 pacientes foi de 38 meses, variando de quatro a 109 meses.

Entre as 90 pacientes que apresentavam NIC I ou lesão menos importante à avaliação inicial, não houve nenhuma evolução para NIC III.

\section{Discussão}

A maior limitação deste estudo foi o fato de ser uma descrição de série de casos, não contando com um grupo de controle. A relevância do estudo se deve ao fato de estes casos terem sido avaliados, tratados e seguidos em um contexto não usual, ou seja, uma UBS. Idealmente, deveríamos comparar os resultados com os de uma população semelhante atendida em uma unidade de referência. Porém, do ponto de vista prático, teríamos grande dificuldade de viabilizar a organização deste grupo de controle. Outra limitação do estudo foi o pequeno tamanho da amostra, pois a unidade não é referência regional para esse tipo de atendimento, atendendo apenas pacientes da área de abrangência da unidade e regularmente matriculadas.

Porém, como o tempo médio de seguimento foi razoavelmente longo (38 meses), e em apenas duas pacientes se constatou evolução para NIC III (1,96\% das que permaneceram na unidade) e nenhuma evolução para carcinoma invasor, e ainda, levando-se em conta que essas duas pacientes foram tratadas na unidade de referência e estão bem, podemos supor que este tipo de atendimento pode ser feito em uma UBS com segurança. Das 115 pacientes da série, 102 (88,7\%) permaneceram na unidade, além das pacientes com resultados citológicos de ASCUS ou HPV que não apresentaram NIC à biópsia (cerca de 50) e que, como foi explicado no Método, não foram incluídas na série. Acreditamos que os resultados não seriam fundamentalmente diferentes em uma unidade de referência. Além disso, os recursos necessários para a realização deste tipo de atendimento, quais sejam, consultório ginecológico, colposcópio e retaguarda para exame anátomo patológico, não são caros nem difíceis de conseguir. Grande parte dos ginecologistas tem treinamento em colposcopia, e caso não tenham, é um treinamento relativamente fácil de se obter. Além de desonerar os serviços de referência, usualmente sobrecarregados, a viabilização deste tipo de atendimento em uma UBS teria outras vantagens em potencial. Acreditamos, por exemplo, que a adesão ao tratamento em uma unidade básica seja maior, devido a aspectos como proximidade da residência, possibilidade de utilizar a unidade para outras necessidades e vínculo com a equipe de saúde ${ }^{20}$. Também na unidade básica, por haver maior proximidade e envolvimento com a população atendida; é mais fácil realizar atividades de vigilância à saúde como, por exemplo, o contato telefônico ou a visita domiciliar para convocação de pacientes faltosas. Várias pacientes da série foram recuperadas ao seguimento por convocação após falta. Acreditamos que o sistema de vigilância, como descrito acima, auxilia no cumprimento dos programas de atenção, e que também subsidia avaliações sistemáticas do trabalho. 
Devemos, porém, chamar a atenção para o fato de que nesta unidade o ginecologista/obstetra não atende toda a demanda da especialidade, sendo que o médico generalista e a enfermagem se encarregam da parcela menos especializada do atendimento de saúde da mulher. Portanto, o atendimento de pacientes com citologia alterada não significa, a princípio, uma sobrecarga de trabalho, e sim a concentração do especialista numa área em que o generalista teria dificuldade em atuar.

Levando em conta o resultado obtido neste período de dez anos, decidimos, a partir de junho de 2006, aumentar o intervalo de retorno de algumas pacientes. Isto tem sido feito de modo individualizado. Naquelas pacientes que desde o início apresentaram colposcopia normal ou biópsia mostrando cervicite, e naquelas cuja biópsia mostrou NIC I e nos últimos quatro ou cinco retornos têm apresentado resultado normal, temos proposto retorno anual. Pretendemos, em breve, propor a volta à citologia trianual, que se propõe para as demais usuárias da unidade nas pacientes com tempo maior de seguimento com as características descritas acima.

Nesta série, não utilizamos tipagem viral para HPV, e parece que tal recurso não traria benefícios importantes no seguimento rotineiro de pacientes em nosso contexto de trabalho.

$\mathrm{Na}$ rotina de atendimento estabelecida pelo programa, realizamos colposcopia em todas as pacientes nas quais se encontra sinais sugesti- vos de infecção por HPV à citologia de Papanicolau. Vale ressaltar que nenhuma paciente da série foi originada deste grupo, ou seja, a colposcopia rotineira com biópsia, quando necessária, nessas pacientes não detectou nenhum caso de neoplasia intra-epitelial cervical.

Vários autores recomendam que se facilite o acesso à colposcopia para a avaliação das alterações citológicas, mesmo as mais leves, devido à possibilidade não desprezível de haver uma alteração maior do que a citologia indica, confirmando dados da literatura ${ }^{21-25}$. Nossos resultados são coerentes com esta recomendação. Como já citado, de 13 pacientes com NIC III confirmado à histologia, apenas três (23\%) tinham citologia concordante, sendo que as demais tinham citologia sugestiva de lesões menos graves. Uma limitação do estudo foi o fato de, até o final do período no qual os dados foram coletados, o laboratório de citologia não ter empregado a nomenclatura de Bethesda de 2001. deste modo, não pudemos relatar nossa freqüência de lesões de alto grau encontradas nas mulheres com citologia ASC-US.

Os resultados são também concordantes com os relatos da literatura $^{5-7}$ sobre o baixo potencial das lesões de baixo grau, para evolução para lesões de alto grau, e potencialmente para o câncer. De 28 pacientes com NIC I confirmada histologicamente e que foram seguidas na unidade, não houve nenhuma evolução para NIC III, após um seguimento médio de 42,1 meses, sendo que 11 não foram tratadas.

\section{Referências}

1. Richart RM. The natural history of cervical intraepithelial neoplasia. Clin Obstet Gynecol 1967;10:748-84.

2. Richart RM, Barron BA. A follow-up study of patients with cervical dysplasia. Am J Obstet Gynecol 1969;105(3):386-93.

3. Östör AG. Natural history of cervical intraepithelial neoplasia: a critical review. Int J Gynecol Pathol 1993;12(2):186-92.

4. Chenoy R, Billingham L, Irani S, Rollason TP, Luesley DM, Jordan JA. The effect of directed biopsy on the atypical cervical transformation zone: assessed by digital imaging colposcopy. Br J Obstet Gynaecol 1996; 103(5):457-62.

5. Lee SS, Collins RJ, Pun TC, Cheng DK, Ngan HY. Conservative treatment of low grade squamous intraepithelial lesions (LSIL) of the cervix. Int $J$ Gynecol Obstet 1998;60(1):35-40.

6. Falls RK. Spontaneous resolution rate of grade 1 cervical intraephitelial neoplasia in a private practice population. Am J Obstet Gynecol 1999; 181(2):278-82.

7. Cardial MFT. Estudo da evolução da neoplasia intraepitelial cervical grau I em pacientes submetidas à conduta expectante. Tese (Mestrado). Faculdade de Ciências Médicas da Santa Casa de São Paulo. São Paulo: 2001.

8. Bollen LJ, Tjong-A-Hung SP, van der Velden J, Mol BW, Lammes FB, ten Kate FW, et al. Human papillomavirus DNA after treatment of cervical dysplasia: low prevalence in normal cytologic smears. Cancer 1996; 77(12):2538-43
9. Londesborough P, Ho L, Terry G, Cuzick J, Wheeler C, Singer A. Human papillomavirus genotype as a predictor of persistence and development of high-grade lesions in women with minor cervical abnormalities. Int $J$ Cancer 1996;69(5):364-8.

10. Hording U, Junge J, Rygaard C, Lundvall F. Management of low-grade CIN: follow-up or treatment? Eur J Obstet Gynecol Reprod Biol 1995; 62(1):49-52.

11. Campion MJ, McCance DJ, Cuzick J, Singer A. Progressive potential of mild cervical atypia: prospective cytlogical, colposcopic, and virological study. Lancet 1986;2(8501):237-40.

12. Kirby TO, Huh WK, Partridge EE. Human papillomavirus triage of patients with atypical squamous cells of undetermined significance on cervical papanicolau smear. Ann Acad Med Singapore 2003;32:590-6.

13. Pfenninger JL. Colposcopy in a family practice residency. The first 200 cases. J Fam Pract 1992;34(1):67-72.

14. Callaway P, Frisch L. Does a family physician who offers colposcopy and LEEP need to refer patients to a gynecologist? J Fam Pract 2000; 49(6):534-6

15. Ministério da Saúde. Instituto Nacional do Câncer. Programa Nacional de Controle do Câncer do Colo do Útero e de Mama - Viva Mulher. Brasília: 2006. Disponivel em URL: http://www.inca.gov.br/conteudo_view.asp? $\mathrm{id}=140$. Acessado em 16 de março de 2006.

16. Coldman A, Phillips N, Kan L, Matisic J, Benedet L, Towers L. Risk of invasive cervical cancer after three consecutive negative Pap smears. J Med Screen 2003;10(4):196-200. 
17. Solomon D, Davey D, Kurman R, Moriarty A, O'Connor D, Prey M, et al. The 2001 Bethesda System: terminology for reporting results of cervical cytology. JAMA 2002;287(16):2114-9.

18. Andrade JM. 0 diagnóstico de células escamosas atípicas em citologia oncológica cervical. Rev Bras Ginecol Obstet 2006;28(2):71-4.

19. Machado-Junior LC. Revisão da literatura médica sobre as evidências de benefícios do tratamento da ectopia do colo do útero. Tese (Mestrado). Faculdade de Medicina da Universidade de São Paulo. São Paulo: 2004.

20. Mckee MD, Schechter C, Burton W, Mulvihill M. Predictors of follow-up of atypical and ASCUS papanicolaou tests in a high-risk population. J Fam Pract 2001;50(7):609.

21. Bolger BS, Lewis BV. A prospective study of colposcopy in women with mild dyskaryosis or koilocytosis. British J Obstet Gynecol 1988;95(11):1117-9.
22. Baldauf JJ, Ritter J. Comparison of the risks of cytologic surveillance of women with atypical cells or low-grade abnormalities on cervical smear: review of the literature. Eur J Obstet Gynecol Reprod Biol 1998;76(2):193-9.

23. Guimarães EM, Pires J, Pelá LB, Aguiar RSF. Evolução de displasia leve diagnosticada por citopatologia: análise retrospectiva de 155 casos. J Bras Patol 1999;35(3):136-40.

24. Lonky NM, Sadeghi M, Tsadik GW, Petitti D. The clinical significance of the poor correlation of cervical dysplasia and cervical malignancy with referral cytologic results. Am J Abstet Gynecol 1999;181(3):560-6.

25. Fairman A, Tan J, Quinn M. Women with low-grade abnormalities on Pap smear should be referred for colposcopy. Aust N Z J Obstet Gynaecol 2004;44(3):252-5 